\title{
Study on the giant positive magnetoresistance and Hall effect in ultrathin graphite flakes
}

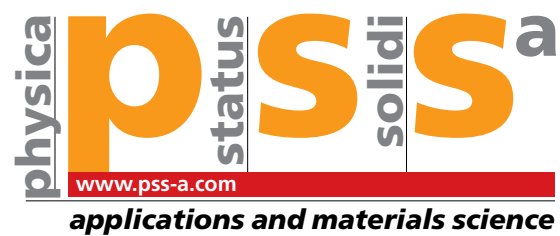

\author{
Rob Vansweevelt', Vincent Mortet', Jan D'Haen ${ }^{1,2}$, Bart Ruttens ${ }^{1,2}$, Chris Van Haesendonck ${ }^{3}$, \\ Bart Partoens ${ }^{4}$, François M. Peeters ${ }^{4}$, and Patrick Wagner ${ }^{*, 1,2}$ \\ ${ }^{1}$ Institute for Materials Research, Hasselt University, Wetenschapspark 1, 3590 Diepenbeek, Belgium \\ ${ }^{2}$ IMEC vzw, Division IMOMEC, Wetenschapspark 1, 3590 Diepenbeek, Belgium \\ ${ }^{3}$ Laboratory of Solid State Physics and Magnetism, Katholieke Universiteit Leuven, Celestijnenlaan 200 D, 3001 Leuven, Belgium \\ ${ }^{4}$ Physics Department - Condensed Matter Theory, Antwerp University, Groenenborgerlaan 171, 2020 Antwerp, Belgium
}

Received 5 November 2010, revised 26 November 2010, accepted 2 February 2011

Published online 25 February 2011

Keywords graphite, Hall effect, magnetoresistance

*Corresponding author: e-mail patrick.wagner@uhasselt.be, Phone: +32 11 268895, Fax: +32 11268899.

In this paper, we report on the electronic transport properties of mesoscopic, ultrathin graphite flakes with a thickness corresponding to a stack of 150 graphene layers. The graphite flakes show an unexpectedly strong positive magnetoresistance (PMR) already at room temperature, which scales in good approximation with the square of the magnetic field. Furthermore, we show that the resistivity is unaffected by magnetic fields oriented in plane with the graphene layers. Hall effect measurements indicate that the charge carriers are p-type and their concentration increases with increasing temperature while the mobility is decreasing. The Hall voltage is non-linear in higher magnetic fields. Possible origins of the observed effects are discussed.

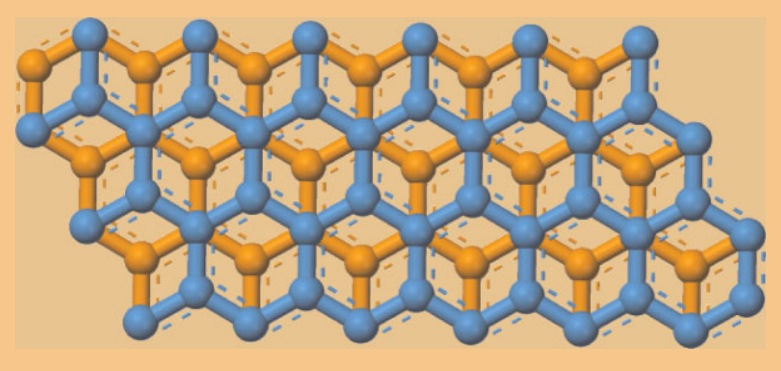

Ball and stick model of the two topmost carbon layers of the hexagonal graphite structure.
1 Introduction Carbon-based materials with $\mathrm{sp}^{2}$ bonding such as graphene, carbon nanowalls, and carbon nanotubes receive since years considerable scientific interest due to their extraordinary electronic transport properties. This has recently culminated in awarding the Nobel Prize in Physics 2010 to Geim and Novoselov for their "groundbreaking experiments regarding the two-dimensional material graphene." The unique features of graphene include the fact that the charge carriers behave like massless Dirac Fermions and the conductivity is not vanishing in the limit of vanishing carrier density [1], the sign of the carriers can be inverted by electric field effects [2], and the quantum Hall effect is observed [3]. Especially interesting with respect to sensor applications are the tunability of the carrier type and concentration as well as the ballistic charge transport with mean free path lengths of several micron. In combination with the extreme surface-to-volume ratio, the electronic band structure and transport properties respond with high sensitivity to various adsorbates (physisorbed gas molecules) as shown in recent work by Leenaerts et al. [4, 5]. The mechanism is based on charge transfer between the adsorbates and the graphene sheets and is expected to fade out in graphite samples with ten or more stacked graphene layers. The influence of covalently bound molecules on the transport in graphene is not yet documented in literature, but first steps have been made with the covalent linking of DNA to carbon nanowalls consisting of four to six graphene layers [6].

In this paper, we focus on the potential of graphene stacks (i.e., ultrathin graphite layers) for magnetic-field 
sensors based on the magnetoresistance effect. Very recent results by Barzola-Quiquia et al. indicate a resistance increase by about $15 \%$ at $270 \mathrm{~K}$ in a field of just $1 \mathrm{~T}$ and the effect becomes even much more pronounced when increasing the magnetic field and lowering the temperature [7]. The positive magnetoresistance (PMR) in pure metals is typically below $5 \%$ in fields of $10 \mathrm{~T}$ at liquid-helium temperature and this has no technological relevance for fieldsensing applications or the readout of magnetically stored data. Instead, magnetic readheads are nowadays based on the giant negative magnetoresistance (GMR) achieved with metallic magnetic/non-magnetic multilayers $[8,9]$. The colossal negative magnetoresistance (CMR) effect in mixedvalency manganese perovskites offers even more substantial resistivity changes (orders of magnitude), but the effect requires fields much higher than those of technical relevance $[10,11]$. Also, materials with a strongly pronounced positive magnetoresistance "PMR" such as $\mathrm{Cr} / \mathrm{Ag} / \mathrm{Cr}$ trilayers [12] and synthetic, boron-doped nano-crystalline diamond [13] show their giant PMR only in fields of several Tesla and at temperatures below $100 \mathrm{~K}$. Therefore, graphite as a nonengineered, natural, and inexpensive material deserves a closer consideration for field-sensing purposes. Due to a wide variability of the material parameters documented in literature (presumably related to impurities and structural defects), we include also Hall-effect measurements in this study to obtain reliable information on the density and mobility of the charge carriers.

\section{Experimental}

2.1 Sample preparation Ultrathin graphite flakes were produced by mechanical exfoliation of highly oriented pyrolytic graphite (HOPG) according to the method described by Geim and Novoselov [14]. Hereby, thin layers of graphite are peeled off from HOPG crystals (SPI-2, SPI Supplies, West Chester, USA) using VD 8 ELEPHOLDER dicing tape. By repeatedly folding and unfolding of the tape with flakes, they become progressively thinner. Ultrathin graphite flakes were transferred to a $\mathrm{Si}$ substrate with a $300 \mathrm{~nm}$ thick, insulating oxide layer by pressing the tape onto the substrate and gently removing the tape. These flakes stick rather tightly to the surface due to van der Waals forces [2]. Next, Au alignment markers were deposited on the $\mathrm{Si}$ substrate with standard photolithography, followed by thermal evaporation of gold, and lift off. The alignment markers serve to identify the position of flakes on the substrate. Suitable flakes with a rectangular shape and typical lateral dimensions of $5 \mu \mathrm{m}$ were selected by optical microscopy.

Before further processing, the samples were annealed in high vacuum of $10^{-7} \mathrm{hPa}$ at $150^{\circ} \mathrm{C}$ during $2 \mathrm{~h}$ to remove adsorbates and tape residues. The actual electrical contact lines and contact pads for wirebonding were written with e-beam lithography. Therefore, a double layer of e-beam resist was spincoated on top of the sample. The resist concentrations and spinning parameters were chosen to yield bottom- and top-resist layers with approximate thicknesses of 300 and $100 \mathrm{~nm}$, respectively. The bottom layer consisted of MMA(8.5)MAA and the top layer of PMMA, both from MicroChem, Newton, USA. The contact pattern with four leads in van der Pauw geometry was designed on basis of earlier taken SEM images of the flake. Subsequently, the pattern was written using the alignment markers and a dose of $270 \mu \mathrm{C} / \mathrm{cm}^{2}$. In order to develop the exposed resist, MIBK developer (MicroChem, Newton) was used in a 1:3 dilution with isopropyl alcohol. Titanium/aluminum contacts were prepared by sputtering a $5 \mathrm{~nm}$ thin layer of Ti followed by thermal evaporation of $70 \mathrm{~nm}$ of Al. Finally, a lift-off step was performed in boiling acetone to remove superfluous metal and e-beam resist. To minimize mechanical strain at the metal-graphite contacts and to remove adsorbates from the resists and developers, a second thermal annealing was done with the same parameters as specified above. Relying on curent-voltage characteristics we have verified that these contacts have Ohmic behavior. A graphite sample, prepared and contacted with the described procedures, is shown in Fig. 1.

2.2 Electrical transport The electronic transport properties were studied for two specific samples using the van der Pauw method [15]: Sample G1 had lateral dimensions of 9.5 by $5 \mu \mathrm{m}^{2}$ with a thickness of $50 \mathrm{~nm}$, corresponding to a stack of 150 graphene layers. From the viewpoint of the electronic band structure this sample should therefore reflect the properties of graphite rather than the features of graphene $[4,5]$. Unless stated otherwise, all data discussed within this paper refer to G1. For comparison, a second sample, G2, was analyzed with lateral dimensions of 8.5 by $9 \mu \mathrm{m}^{2}$ and a thickness of $25 \mathrm{~nm}$, corresponding to 75 graphene layers. The sample thicknesses were determined by atomic force microscopy after performing the transport measurements. The contacted samples were fixed on a holder on the cold finger of a flow cryostat (MicroStat, Oxford Instruments, UK) with a maximum temperature range from

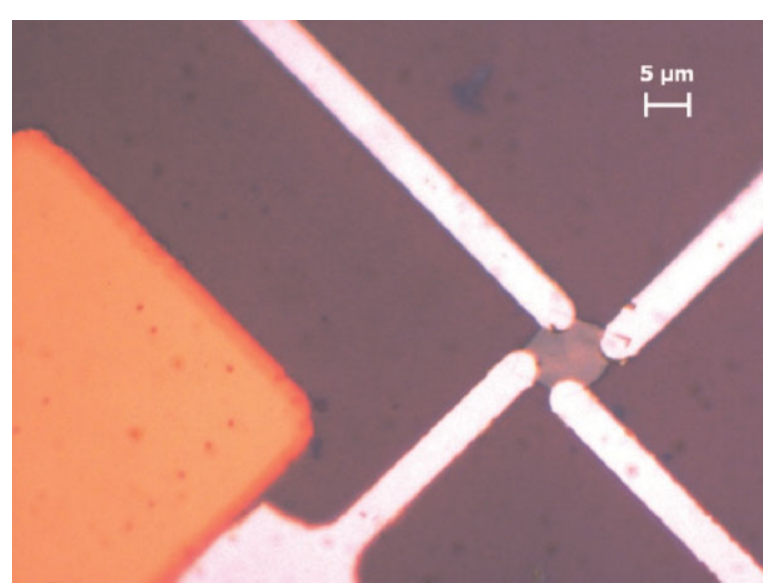

Figure 1 (online colour at: www.pss-a.com) Optical image of an ultrathin graphite flake with four $\mathrm{Ti} / \mathrm{Al}$ electrical contact lines made with e-beam lithography. The structure at the left lower corner is a $\mathrm{Au}$ alignment marker. 
4.2 to $400 \mathrm{~K}$. Electrical connections were obtained by wirebonds between the sample and the holder, and with triaxial cables between the cryostat and all external devices. The current source, Keithley 6221, was operated at a level of $50 \mu \mathrm{A}$ while voltages were measured with a Keithley 2400 source meter. To allow for interchanging the current- and the voltage probes according to the van der Pauw method, we employed a grounded and shielded, manually operated switchbox, taking care that the current source was set to zero during the switching steps.

Magnetic fields, oriented parallel to the c-axis of the graphite samples, were generated with an electromagnet, type Bruker B-E $15 \mathrm{v}$. The relationship between the coil current and the resulting field at the samples position was calibrated with a Hall sensor, LakeShore HGT-2010, revealing field strengths up to a maximum of $2.07 \mathrm{~T}$. In the geometry with B parallel to the c-axis of the graphite flakes, we measured sheet resistivities (transverse magnetoresistance) and Hall voltages for temperatures between 77 and $300 \mathrm{~K}(\mathrm{G} 1)$, and between 4.2 and $400 \mathrm{~K}(\mathrm{G} 2)$. Furthermore, magnetotransport studies were performed with several angles $\theta$ between the $B$ field and the c-axis to evaluate whether there is also a noticeable longitudinal contribution to the magnetoresistance.

\section{Results and discussion}

3.1 Longitudinal resistivity As shown in Fig. 2, the in-plane room temperature resistivity of the graphite flake is $40 \mu \Omega \mathrm{cm}$. This is by a factor of 20 smaller than literature values on bulk samples of graphite. Therefore, the resistivity of these small-volume samples appears to be intrinsic and not dominated by grain-boundary contributions. Although the temperature dependence of the zero-field resistivity (insert of Fig. 2) is apparently thermally activated, it cannot be easily described by an Arrhenius law or hopping models in two or three dimensions.

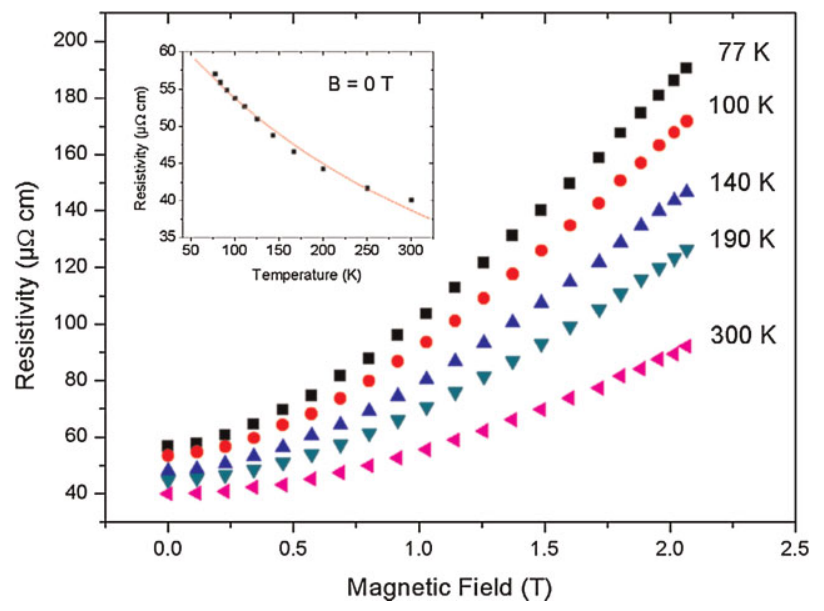

Figure 2 (online colour at: www.pss-a.com) Magnetoresistance behavior of sample G1 at selected temperatures between 77 and $300 \mathrm{~K}$. The magnetic field is oriented parallel to the $c$ axis. The zero field resistivity is given in the insert with the solid line corresponding to the fit function described in the text.
Refinements, taking into account temperature-dependent pre-factors, do also not allow bringing the experimental data in agreement with theoretical models. However, an empirical expression, which will further be addressed in Section 3.2, is in very good agreement with the data:

$$
\rho(T)=\frac{\rho(T \rightarrow 0)}{1+\alpha \cdot T} .
$$

For the fit parameters, we obtain the numerical values $\rho(T \rightarrow 0)=66.8 \mu \Omega \mathrm{cm}$ and $\alpha=2.42 \times 10^{-3} \mathrm{~K}^{-1}$ with $R^{2}=0.98\left(R^{2}\right.$ is the coefficient of determination). Applying the same function to sample G2 gave $\rho(T \rightarrow 0)=$ $93.1 \mu \Omega \mathrm{cm}$ and $\alpha=1.98 \times 10^{-3} \mathrm{~K}^{-1}$ with $R^{2}=0.91$.

The PMR is strongly pronounced already at room temperature and in moderate fields. For a quantitative description we define a PMR ratio as follows:

$$
\operatorname{PMR}=\frac{\Delta \rho}{\rho_{0}}=\frac{\rho(B)-\rho(B=0)}{\rho(B=0)} .
$$

In a field of $2 \mathrm{~T}$, the PMR exceeds $100 \%$ at $300 \mathrm{~K}$ and $300 \%$ at $77 \mathrm{~K}$ and the magnitude of the effect as such is in good agreement with the work by Barzola-Quiquia et al. [7]. Note that in comparison to pure metals, the PMR is substantial and easily measurable, it occurs already at room temperature, and the magnetic-field scale is moderate albeit not yet sufficiently low for the readout of magnetically stored data. Next, we analyzed the field dependence of the PMR magnitude for the different temperatures under study. Metallic-like conductors fulfill the classical Kohler rule [16], stating that the relative resistivity increase is a universal function of the ratio between the magnetic field $\mathrm{B}$ and the zero-field resistivity [Eq. (3a)]. This originates from the fact that, under the influence of a magnetic field, the charge carriers will move on fragments of Landau circles rather than along straight lines. In turn, this reduces their effective mean free path.

In non-magnetic, clean metals and small magnetic fields, the function $f$ is strictly quadratic in $\mathrm{B}$, resulting in a more specific representation of Kohler's rule [Eq. (3b)]:

$$
\frac{\Delta \rho}{\rho_{0}} \propto f\left(\frac{B}{\rho_{0}}\right) \Rightarrow \Delta \rho \cdot \rho_{0} \propto B^{2} .
$$

A scaling in the sense of Eq. (3b) should collapse all $\rho(B, T)$ data on one single curve. Representing the data given in Fig. 2 according to Eq. (3b) results in the magnetoresistance traces shown in Fig. 3: noteworthy, this scaling deviates from $\Delta \rho \propto B^{2}$, which holds in non-magnetic, metallic conductors, and follows rather a power law relationship $\Delta \rho \propto B^{\beta}$ with a weakly temperature dependent exponent $\beta$, ranging from $1.51(77 \mathrm{~K})$ to $1.72(300 \mathrm{~K})$. The reliability coefficients for all these temperatures are above $R^{2}=0.99$. Also, there is a systematic displacement along the $\Delta \rho \cdot \rho_{0}$ axis, which can be understood if the density of the charge carriers itself is temperature dependent. This aspect will be further addressed in Section 3.2 below that deals with 


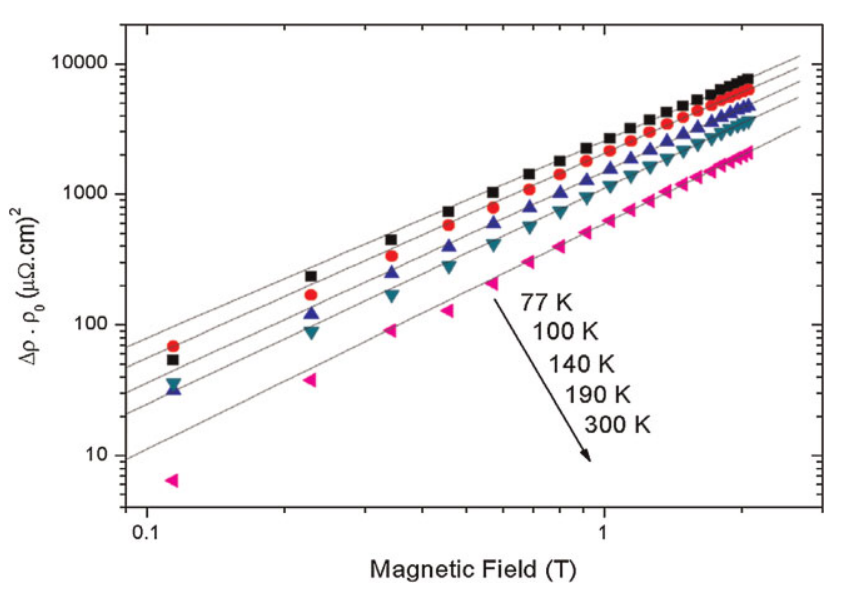

Figure 3 (online colour at: www.pss-a.com) Magnetoresistance behavior of sample G1 plotted according to Kohler's rule for metals. There is a systematic, temperature-dependent displacement and the fitted slopes (ranging from 1.5 to 1.7 ) are smaller than the expected $B^{2}$ dependence.

the Hall-effect measurements. Certain deviations from Kohler's rule have actually been reported for many metallike materials, including layered organic metals [17] and cuprate superconductors in their normal state [18].

The fact that $\beta<2$ may possibly be interpreted in terms of the presence of correlated magnetic impurities or other local magnetic moments. At least in the ferromagnetic CMR materials, the magnetoresistance is known to be linear in the magnetic field $(\beta=1)$ as long as the magnetization is below the saturation limit [11]. In our case, the impurity concentration of the HOPG samples is below $10 \mathrm{ppm}$ [19], which sets a maximum on the concentration of (magnetic) atoms or ions. A careful analysis by X-ray photoemission spectroscopy gave a negative result with respect to the presence of iron or other magnetic impurities. Although the concentration of $10 \mathrm{ppm}$ is low in absolute terms, the average distance between these dopants or impurities would correspond to $200 \mathrm{~nm}$, meaning that scattering centers cannot be ignored. Irrespective of whether there are magnetic ions in the graphite lattice (e.g., in an intercalated way) ferromagnetic moments have also been reported in nominally iron-free HOPG samples and were attributed to topological defects or electron-electron correlations [20]. The recently discovered ferromagnetism of hydrogen vacancies in graphane, see Ref. [21], should be negligible in our context due to the almost bulk character of the graphite flakes as compared to few-layer graphene.

In order to check whether there is also a longitudinal magnetoresistive effect (field oriented in plane with the graphene sheets), we studied the magnetoresistance for several angles $\theta$ between the field direction and the c-axis of the graphite flakes. These measurements, illustrated in Fig. 4, were performed at $300 \mathrm{~K}$ in the maximum available field of $2.07 \mathrm{~T}$. There is neither a PMR nor a negative magnetoresistance measurable when the field is precisely in plane and the PMR is maximal when $B$ is parallel to the

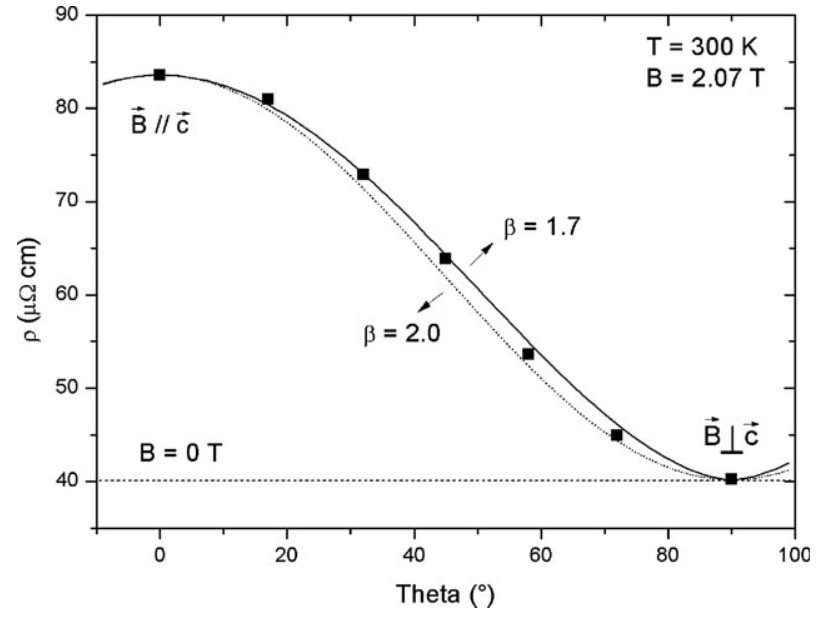

Figure 4 Magnetoresistance behavior of sample G1 plotted at a fixed temperature and magnetic field for various angles between the field direction and the graphene sheets. The fit curves show that the perpendicular field component is responsible for the PMR effect.

$c$-axis of the flakes. The relevant field component is the component parallel to the $c$-axis of the graphene sheets, which can be expressed by the following scaling relation between the PMR amplitude and the angle $\theta$ :

$$
\rho(B, \theta)=\rho(B=0)+\Delta \rho(B) \cos ^{\beta} \theta .
$$

Within the precision of our measurements, in-plane components of the magnetic field have no effect, which is similar to other quasi two-dimensional materials such as the high-temperature superconductors [22]. Note that the curves presented in Fig. 4 have no free fitting parameters, the only input data are $\rho\left(2.07 \mathrm{~T}, \theta=90^{\circ}\right) \approx \rho(0 \mathrm{~T})=40.2 \mu \Omega \mathrm{cm}$ and $\rho\left(2.07 \mathrm{~T}, \theta=0^{\circ}\right)=83.6 \mu \Omega \mathrm{cm}$. The dashed line, obtained with $\beta=2$ (non-magnetic materials) is already in good agreement with the data. An improved correspondence is obtained with $\beta=1.7$, consistent with the results in Fig. 3 .

3.2 Hall effect Based on the van der Pauw measurements, we extracted also the temperature and field dependence of the Hall voltage, which is used as an input parameter for the determination of charge-carrier concentrations and mobilities. Since the van der Pauw scheme is more susceptible to potential geometrical and electronic errors than a Hall-bar configuration, we first verified the linearity of the Hall signal with respect to the magnetic field and the current at $300 \mathrm{~K}$. As shown in Fig. 5, both linearities are fulfilled, giving confidence in the reliability of the consecutively performed low-temperature measurements. From the sign of the Hall voltage $U_{\mathrm{H}}$, we conclude that the dominant charge carriers are p-type.

The relationship between the Hall coefficient $R_{\mathrm{H}}$, the Hall voltage $U_{\mathrm{H}}$, the current $I$, and the sample thickness $d$ and 


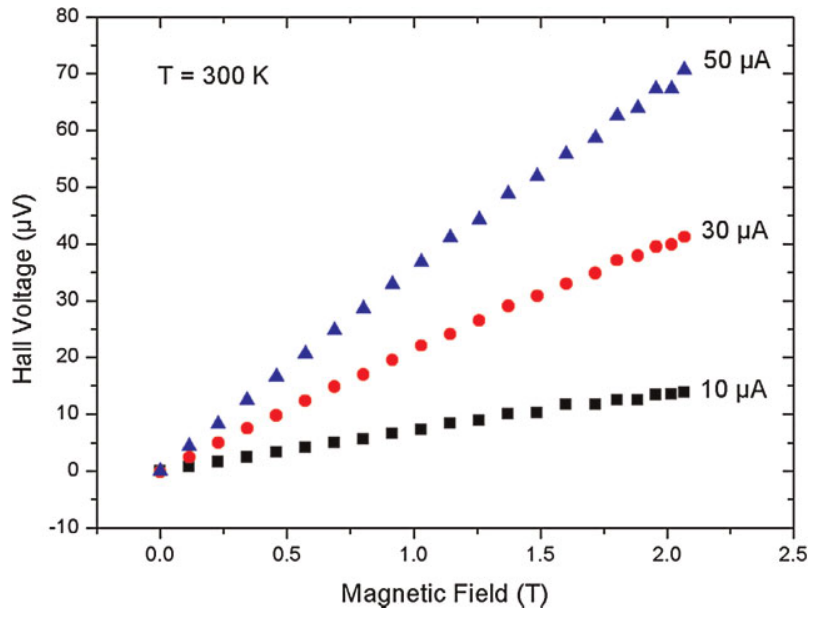

Figure 5 (online colour at: www.pss-a.com) The Hall voltage at $300 \mathrm{~K}$ increases strictly linearly with the applied magnetic field and varies proportional to the measuring current.

carrier concentration $n$ is given by:

$$
R_{\mathrm{H}}=\frac{U_{\mathrm{H}} d}{I B}=\frac{1}{n e} .
$$

Here, we assume a one-band model with p-type carriers only and e represents a positive elemental charge. For a field $B=2.0 \mathrm{~T}$, the given sample thickness $d=50 \mathrm{~nm}$, and the three different currents $(10,30$, and $50 \mu \mathrm{A})$, we obtain $R_{\mathrm{H}}=3.5( \pm 0.2) \times 10^{-8} \mathrm{~m}^{3} / \mathrm{C}$. This corresponds to a carrier density $n=1.7( \pm 0.1) \times 10^{26}$ holes $/ \mathrm{m}^{3}$. The concentration of carbon atoms in single-crystalline graphite is $1.13 \times 10^{29} \mathrm{~m}^{-3}$ and therefore we have an approximate ratio of 1 mobile charge carrier per 660 carbon atoms. According to the semi metallic behavior of graphite, a more refined analysis can be based on a two-band model with p- and ntype carriers [23]. However, this is less straightforward because it requires the mobility ratio between $\mathrm{p}$ - and n-type carriers as an additional, unknown parameter.

In the following, we studied the field dependence of the Hall voltage also for selected temperatures between 77 and $300 \mathrm{~K}$, see Fig. 6. The current in these measurements was always set to $50 \mu \mathrm{A}$. Above $1 \mathrm{~T}$, there is a clear downward bend of the $U_{\mathrm{H}}(B)$ curves, which cannot be explained within the one-band model. Moreover, this is in contrast to earlier Hall studies on graphite by Kopelevich et al., where an upward bent of the Hall-voltage traces was observed and explained by a field-induced exciton-pairing mechanism [24]. A control experiment on sample G2 has confirmed the downward bent: the initial slope of the $U_{\mathrm{H}}(B)$ traces at $300 \mathrm{~K}$ was also positive, but $U_{\mathrm{H}}$ even became zero around $1.5 \mathrm{~T}$ and turned out to have a negative sign for higher fields.

There are three possible explanations, but the given dataset with its limited field- and temperature range does not yet allow making a clear distinction: (i) signatures of the quantum-Hall effect are one possibility, but quantum oscillations in graphite and few-layer graphene have until

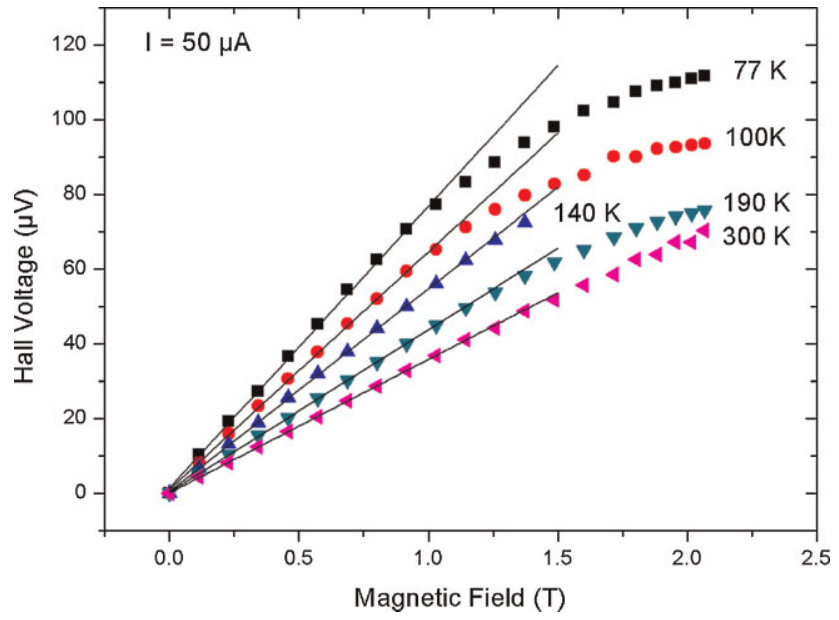

Figure 6 (online colour at: www.pss-a.com) Hall voltage versus magnetic field for selected temperatures between 77 and $300 \mathrm{~K}$. For fields above $1 \mathrm{~T}$ there is a deviation from linear behavior, suggesting the presence of anomalous contributions.

now only been observed for temperatures below $50 \mathrm{~K}$ and $2 \mathrm{~T}$ is an unusually low field scale [25-27]. (ii) One may also infer a compensated Hall effect with a contribution by electron-type carriers: the non-linear behavior could then indeed be caused by a field-dependent ratio between the hole- and electron mobilities. Compensation effects can therefore bring about apparent changes from hole- to electron type carriers [28]. (iii) Finally, there is an anomalous Hall effect in magnetic metals and oxides with a superposition of the ordinary Hall effect and scattering mechanisms related to the magnetic moments of the samples, see e.g., [29] and references therein. As mentioned above, HOPG graphite is known to contain magnetic moments [20], but their concentration is by orders of magnitude lower than for example in iron or in manganese-based perovskites. Therefore, also the validity of this explanation is unclear and further studies are required, including magnetization measurements, to pinpoint the precise origin of the nonlinear $U_{\mathrm{H}}(B)$ behavior in higher magnetic fields.

To evaluate the temperature dependence of the chargecarrier density and mobility, we limit the data analysis to the low-field regime and calculated $n$ based on the initial slopes of the $U_{\mathrm{H}}(B)$ traces presented in Fig. 6 . The carrier concentrations are given in Fig. 7 and reveal a sublinear increase with temperature, in agreement with the halfmetallic character of graphite. The existence of a bandgap cannot be excluded, but the characteristic energy scale, if any, cannot be extracted from these data. At most, we can conclude that the temperature dependence of $n$ follows approximately a scaling proportional to the square root of temperature. From the zero-field resistivities $\rho_{0}$ and the carrier densities, we finally derived the carrier mobilities by using the formula:

$$
\mu(B \rightarrow 0)=\frac{R_{\mathrm{H}}(B \rightarrow 0)}{\rho_{0}}=\frac{e \tau}{m} .
$$




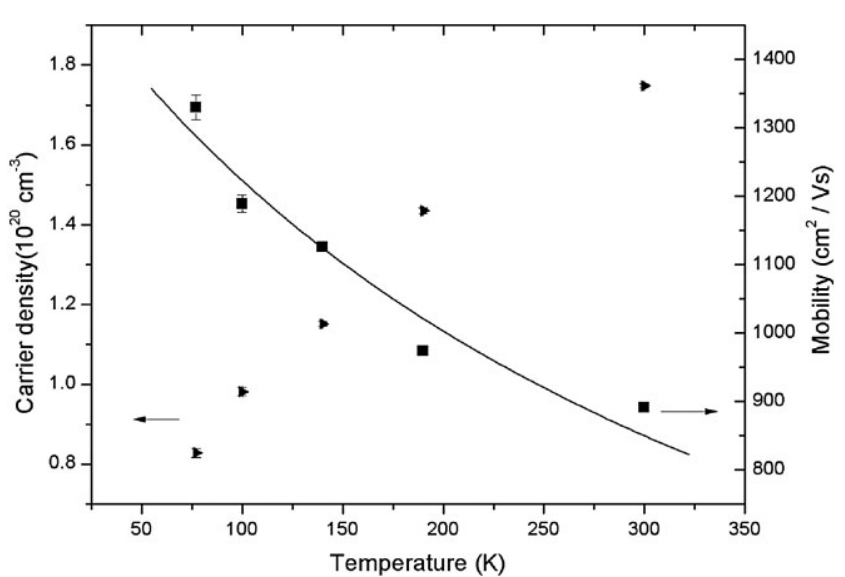

Figure 7 Temperature dependence of the charge-carrier density $n$ (triangles, left scale) and the charge-carrier mobility $\mu$ (squares, right scale), derived in the limit of low magnetic fields $B \rightarrow 0$. The solid line for the mobility scaling was calculated using Eq. (7).

The mobility values $\mu$ are also given in Fig. 7 and $\mu$ turns out to be independent of the absolute carrier concentrations. In a material such as graphite with a low absolute chargecarrier concentration, possible interactions between charge carriers can in good approximation be neglected. Since $\mu$ is related to the charge, effective mass $m$, and scattering time $\tau$, one can derive a temperature dependence based on Matthiessen's rule for superimposed scattering mechanisms.

The scattering due to impurities and grain boundaries has a temperature independent value $\tau_{0}$ and scattering due to phonons follows a scaling according to $\tau_{1} \sim T^{-1}$. The effective scattering time follows the relationship $1 / \tau=1 /$ $\tau_{0}+1 / \tau_{1}$. Therefore, the temperature dependence of the mobility should be given by the expression:

$$
\mu(T)=\frac{\mu(T \rightarrow 0)}{1+\gamma T} .
$$

With the parameters $\mu(T \rightarrow 0)=1566 \mathrm{~cm}^{2} / \mathrm{Vs}$ $\left( \pm 96 \mathrm{~cm}^{2} / \mathrm{Vs}\right)$ and $\gamma=2.8 \times 10^{-3} \mathrm{~K}^{-1}\left( \pm 0.6 \times 10^{-3} \mathrm{~K}^{-1}\right)$ we obtain indeed a nice correspondence with the experimental data, as illustrated in Fig. 7. The $R^{2}$ value of the calculated curve is 0.93 . The absolute mobility values, at room temperature as well as in the low-temperature limit, are high as compared to metals and similar to electronic grade silicon. For comparison, in few-layer graphene sheets with a suitable gate voltage, values up to $6 \times 10^{4} \mathrm{~cm}^{2} / \mathrm{V}$ s have been reported at a temperature of $4 \mathrm{~K}$ [2]. The current record value is $2 \times 10^{5} \mathrm{~cm}^{2} / \mathrm{Vs}$ at $5 \mathrm{~K}$ in gated single-layer graphene sheets, which were suspended to exclude any disturbing influence from an underlying material [30]. Although the value $\mu(T \rightarrow 0) \approx 1566 \mathrm{~cm}^{2} / \mathrm{Vs}$ is reduced with respect to gated few- or single-layer graphene, the mean free path is in the order of $100 \mathrm{~nm}$ to $1 \mu \mathrm{m}$, which suggests a ballistic charge transport. Here, we have assumed that the carrier mass is in the order of the free-electron mass and that the carrier speed is in the range $10^{5}-10^{6} \mathrm{~m} / \mathrm{s}$. The mean free path of $200 \mathrm{~nm}$, estimated on the basis of the maximum impurity concentration in Section 3.1, fits excellently within the margins obtained from the mobility analysis. The temperature dependence of the mobility, given by Eq. (7), suggests that the resistivity increases with increasing temperature. This is obviously not the case (see insert of Fig. 2) because the temperature-induced rise of the charge-carrier density overcompensates the diminishing mobility. An improved description of the experimentally observed resistivity decrease with increasing temperature requires therefore mainly an in-depth understanding of the temperature dependence of the charge-carrier density.

4 Conclusions We have demonstrated that ultrathin graphite layers have an inherent potential for magnetic-field sensors, operating already at room temperature. The resistivity increases markedly at fields below the $1 \mathrm{~T}$ range and the only drawback is the non-linear (close to quadratic, $\beta \approx 1.6 \pm 0.1$ ) response with respect to the applied field. In order to obtain linearized field-response characteristics, it appears recommendable to induce intentionally ferromagnetic dopants or other ferromagnetic correlations associated with specific adsorbates or topological defects. The creation of carbon vacancies by irradiation with high-energetic ions is therefore a promising route to create magnetic moments with a controlled concentration [31, 32]. Also, the Hall signal can be used for field sensing purposes and the preconditions are excellent: graphite is a system with an intrinsically low carrier density - and it can be spliced down to ultralow thicknesses until the limit of a single graphene sheet is reached. Both elements, the limited thickness and the low carrier density, are beneficial to obtain large Hall signals already in low magnetic fields. Hereby, it is obvious that the micromechanical exfoliation of HOPG is not ideally suited for the series production of graphite- or graphene-based Hall sensors. However, there is very recent, highly promising progress to produce electronic-grade graphene on large-area substrates by chemical vapor deposition [33] and by a wetchemical approach in combination with Langmuir-Schaefer deposition [34].

From a more fundamental point of view, we have found that the PMR in ultrathin graphite flakes follows Kohler's rule with the side condition that the charge-carrier density is temperature dependent. The surprisingly strong PMR amplitude is related to the fact that the mean free path, in the order of $100 \mathrm{~nm}$ or more, is in the same range as the radius of Landau circles. The temperature dependence of the chargecarrier mobility can perfectly be understood in terms of scattering at impurities and thermal excitations. In order to determine the charge carrier density, we focused on the low-field limit, where there is a linear relationship between the applied field and the resulting Hall voltage. At higher field, the Hall effect shows anomalous features, which can possibly originate from compensation effects (superposition of hole- and electron carriers) or localized magnetic moments. 
Acknowledgements The authors gratefully acknowledge the support by FWO - Research Foundation Flanders (project G.0159.07 "Structural and electronic properties of biologically modified, graphene-based layers"), by the Federal Belgian Interuniversity Attraction Poles Programme BELSPO (project IAP VI P6/42 "Quantum effects in clusters and nanowires") and by the Methusalem network "NANO - Antwerp-Hasselt," funded by the Flemish Community. Technical assistance by Stoffel D. Janssens (magnet calibration and software development), Dr. Hong Yin (AFM-based thickness studies), Dr. Ronald Thoelen (data analysis), and Prof. Hans-Gerd Boyen (XPS spectroscopy) is greatly appreciated.

\section{References}

[1] K. S. Novoselov, A. K. Geim, S. V. Morozov, D. Jiang, M. I. Katsnelson, I. V. Grigorieva, S. V. Dubonos, and A. A. Virsov, Nature 438, 197-200 (2005).

[2] K. S. Novoselov, A. K. Geim, S. V. Morozov, D. Jiang, Y. Zhang, S. V. Dubonos, I. V. Grigorieva, and A. A. Virsov, Science 306, 666-669 (2004).

[3] Y. Zhang, Y.-W. Tan, H. L. Störmer, and P. Kim, Nature 438, 201-204 (2005).

[4] O. Leenaerts, B. Partoens, and F. M. Peeters, Phys. Rev. B 77, 125416-125423 (2008).

[5] O. Leenaerts, B. Partoens, and F. M. Peeters, Appl. Phys. Lett. 24, 243125-243133 (2008).

[6] R. Vanweevelt, A. Malesevic, M. Van Gompel, A. Vanhulsel, S. Wenmackers, J. D'Haen, V. Vermeeren, M. Ameloot, L. Michiels, C. Van Haesendonck, and P. Wagner, Chem. Phys. Lett. 485, 196-201 (2010).

[7] J. Barzola-Quiquia, J.-L. Yao, P. Rödiger, K. Schindler, and P. Esquinazi, Phys. Status Solidi A 205, 2924-2933 (2008).

[8] M. N. Baibich, J. M. Broto, A. Fert, F. N. Vandau, F. Petroff, P. Eitenne, G. Creuzet, A. Friederich, and J. Chazelas, Phys. Rev. Lett. 61, 2472-2475 (1988).

[9] G. Binasch, P. Grünberg, F. Saurenbach, and W. Zinn, Phys. Rev. B 39, 4828-4830 (1989).

[10] R. Von Helmolt, J. Wecker, B. Holzapfel, L. Schultz, and K. Samwer, Phys. Rev. Lett. 71, 2331-2333 (1993).

[11] P. Wagner, I. Gordon, L. Trappeniers, J. Vanacken, F. Herlach, V. V. Moshchalkov, and Y. Bruynseraede, Phys. Rev. Lett. 81, 3980-3983 (1998).

[12] G. Verbanck, K. Temst, K. Mae, R. Schad, M. J. Van Bael, V. V. Moshchalkov, and Y. Bruynseraede, Appl. Phys. Lett. 70, 1477-1479 (1997).

[13] B. L. Willems, G. Zhang, J. Vanacken, V. V. Moshchalkov, S. D. Janssens, O. A. Williams, K. Haenen, and P. Wagner, J. Appl. Phys. 106, 33711-33715 (2009).

[14] A. K. Geim and K. S. Novoselov, Nature Mater. 6, 183-191 (2007).
[15] L. J. van der Pauw, Philips Res. Rep. 13, 1-9 (1958).

[16] J. M. Ziman, Electrons and Phonons (Clarendon Press, Oxford, England, 1963).

[17] R. H. McKenzie, J. S. Qualls, S. Y. Han, and J. S. Brooks, Phys. Rev. B 57, 11854-11857 (1998).

[18] J. M. Harris, Y. F. Yan, P. Matl, N. P. Ong, P. W. Anderson, T. Kimura, and K. Kitazawa, Phys. Rev. Lett. 75, 1391-1394 (1995).

[19] http://www.2spi.com/catalog/new/hopgsub/Datasheet/php (latest update: Nov. 03, 2010).

[20] P. Esquinazi, A. Setzer, R. Höhne, C. Semmelhack, Y. Kopelevich, D. Spemann, T. Butz, B. Kohlstrunk, and M. Lösche, Phys. Rev. B 66, 24429-24438 (2002).

[21] J. Berashevich and T. Chakraborty, Nanotechnology 21, 355201-355205 (2010).

[22] G. Jakob, M. Schmitt, Th. Kluge, C. Tomé-Rosa, P. Wagner, Th. Hahn, and H. Adrian, Phys. Rev. B 47, 12099-12103 (1993).

[23] B. Partoens and F. M. Peeters, Phys. Rev. B 74, 75404-75414 (2006).

[24] Y. Kopelevich, J. C. Medina Pantoja, R. R. da Silva, F. Mrowka, and P. Esquinazi, Phys. Lett. A 355, 233-236 (2006).

[25] Y. Kopelevic, B. Raquet, M. Goiran, W. Escoffier, R. R. da Silva, J. C. Medina Pantoja, I. A. Luk'yanchuk, A. Sinchenko, and P. Monceau, Phys. Rev. Lett. 103, 116802-116805 (2009).

[26] P. Stamenov, V. Krstic, and J. M. D. Coey, J. Magn. Magn. Mater. 290/291, 1402-1404 (2005).

[27] C. Berger, Z. Song, T. Li, X. Li, A. Y. Ogbasghi, R. Feng, Z. Dai, A. R. Marchenkov, E. H. Conrad, P. N. First, and W. A. de Heer, J. Phys. Chem. B 108, 19912-19916 (2004).

[28] G. Jakob, F. Martin, W. Westerburg, and H. Adrian, Phys. Rev. B 57, 10252-10255 (1998).

[29] P. Wagner, D. Mazilu, L. Trappeniers, V. V. Moshchalkov, and Y. Bruynseraede, Phys. Rev. B 55, 14721-14724 (1997).

[30] K. I. Bolotin, K. J. Sikes, Z. Jiang, M. Klima, G. Fudenberg, J. Hone, P. Kim, and H. L. Störmer, Solid State Commun. 146, 351-355 (2008).

[31] M. M. Ugeda, I. Brihuega, F. Guinea, and J. M. GómezRodríguez, Phys. Rev. Lett. 104, 96804-96807 (2010).

[32] M. A. Ramos, J. Barzola-Quiquia, P. Esquinazi, A. MuñozMartin, A. Climent-Font, and M. García-Hernández, Phys. Rev. B 81, 214404-214419 (2010).

[33] K. S. Kim, Y. Zhao, H. Jang, S. Y. Lee, J. M. Kim, K. S. Kim, J.-H. Ahn, P. Kim, J.-Y. Choi, and B. H. Hong, Nature 457, 706-710 (2009).

[34] R. Y. N. Gengler, A. Veligura, A. Enotiadis, E. K. Diamanti, D. Gournis, C. Józsa, B. J. van Wees, and P. Rudolf, Small 6, 35-39 (2010). 